\section{INCREASING CAPACITY FOR AUTOLOGOUS STEM CELL TRANSPLANTS FOR LYMPHOMAS: A QUALITY IMPROVEMENT STUDY}

Uday Deotare, Adrienne Fulford, Anargyros Xenocostas, Deanna Caldwell, Sue Nugent, Susan Reiger, Mark Mussio, Alan Gob. London Health Sciences Centre, London, ON, Canada

\section{$10.1136 / \mathrm{bmjoq}-2021-\mathrm{IHI} .8$}

Background Autologous Stem Cell Transplant (ASCT) patients have an inpatient length of stay (LOS) of 21-28 days. In a resource constrained environment, this leads to increasing wait times and limited ability to do transplants. We tried to create additional capacity for transplants at our institution by studying the root causes of this problem and designing improvement in our own resource constrained environment.

Objectives Our main objective or Big Dot aim was to increase the capacity of ASCT by $20 \%$ by Dec 2020 (figure 1). The project aim was to decrease the LOS in ASCT patients by 2 days by May 2020.
Methods A major root cause of increased LOS of ASCT patients was identified (figures 2 and 3) and was targeted for improvement. The first PDSA ramps consisted of conversion of IP PowerPlan to OP for administration of Chemotherapy. The second PDSA ramps consisted of creation of a modified CoSTARS assessment tool used to determine the risk pathway for readmissions. The subsequent PDSA ramps consisted of education of In-charge Nurses, trainees and patients. The data of LOS was plotted on a Shewart Process Control Chart "I Chart" using QI Macros software.

Results The median LOS at the baseline was 24.5 days (range: 16-42) (figure 4). After the implementation of the change idea on 10 Dec 2019 till 5 Feb 2021, the LOS for ASCT decreased to 16.5 days (range: 13-21) (figure 5 ). The capacity for ASCT in our institution increased by $40 \%$ during the study period. We were able to save 120 inpatient days for ASCT after change from IP to OP Conditioning Chemotherapy in 15 pts (CoVid- May to Jul 2020).

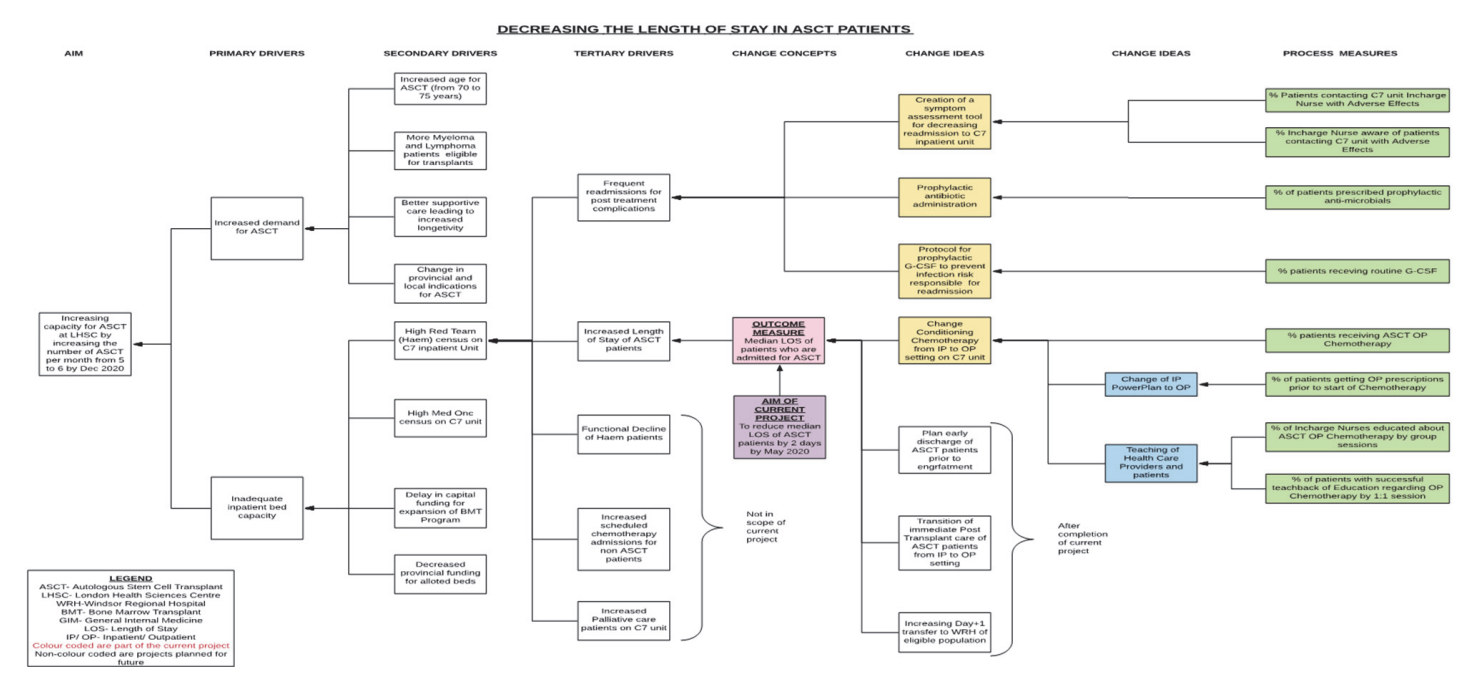

Abstract 8 Figure 1 Driver diagram

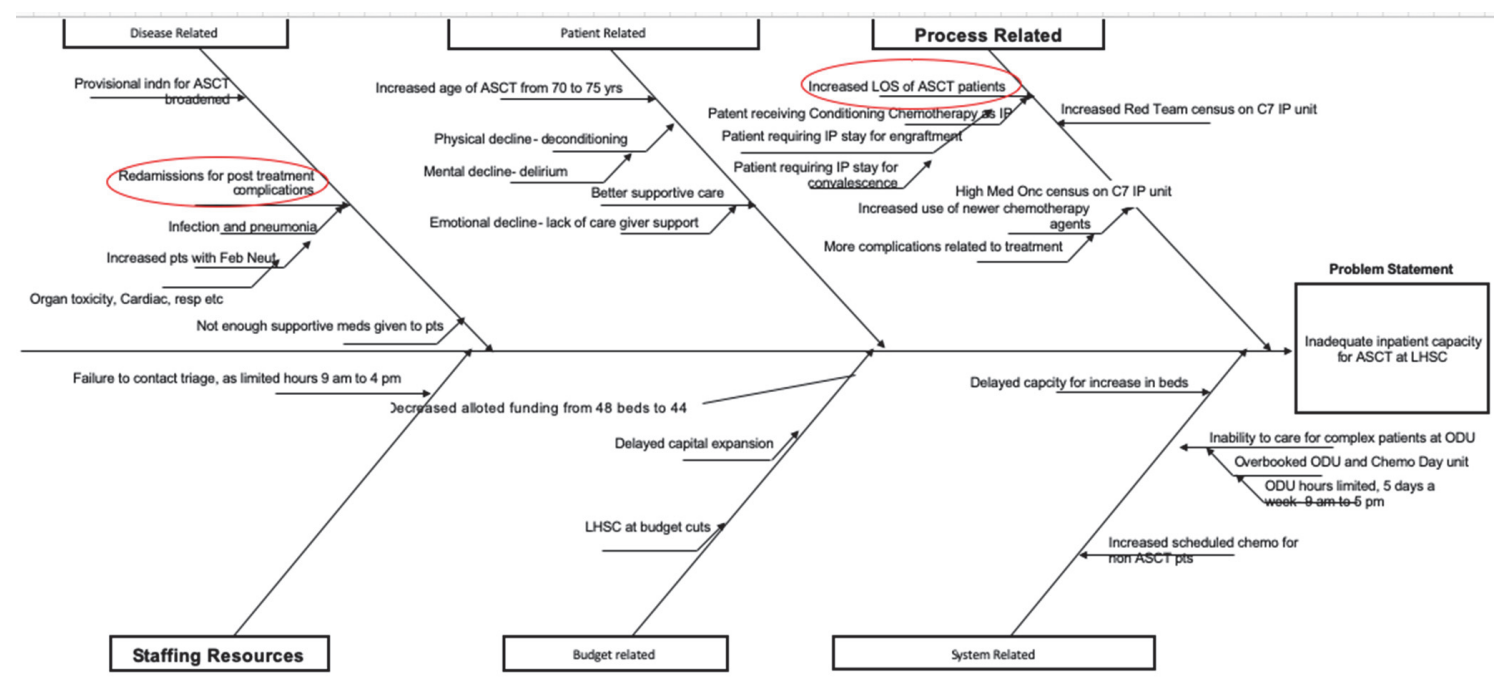

Abstract 8 Figure 2 Ishikawa fishbone diagram 


\section{Frequency of occurrence}
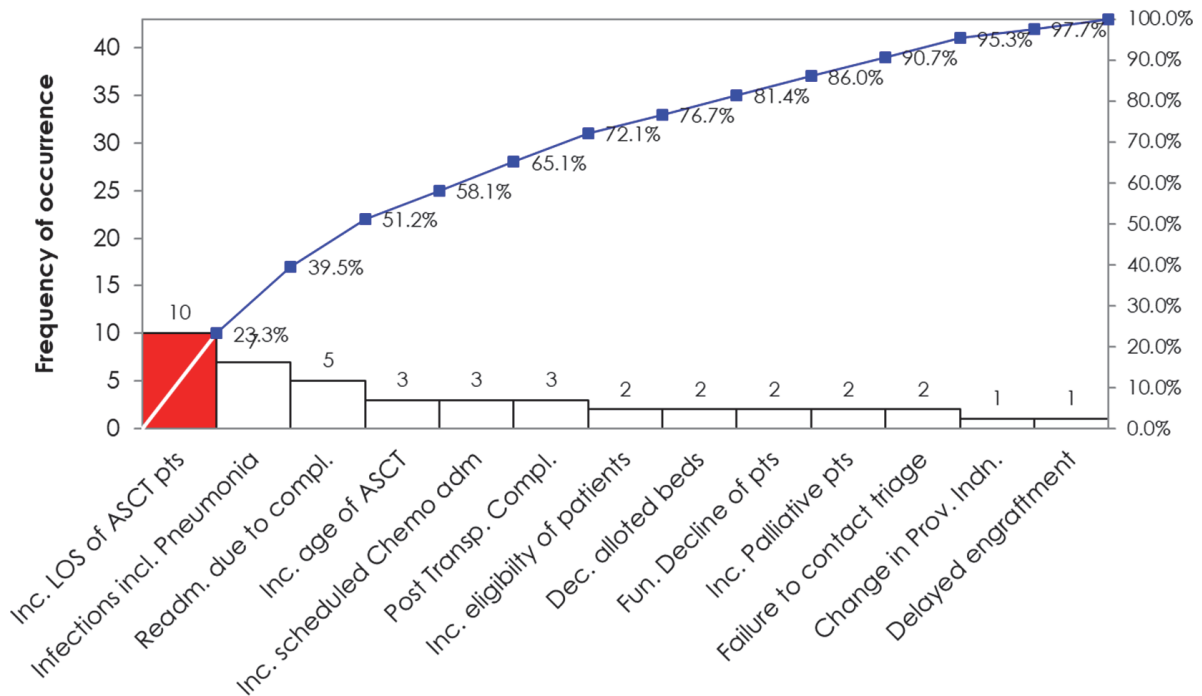

Root Causes of Inc.LOS in Hem C7 pts

Abstract 8 Figure 3 Pareto chart

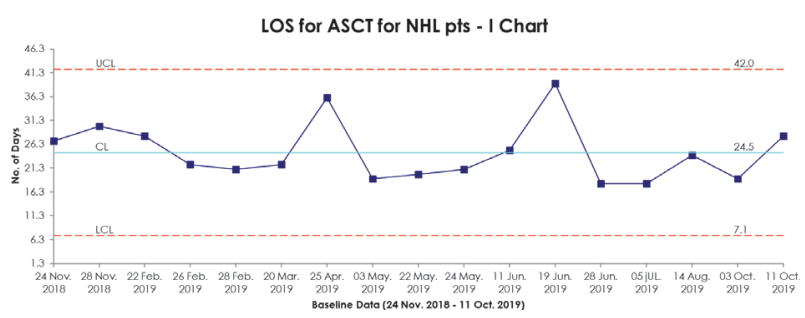

Abstract 8 Figure 4 LOS for ASCT for NHL patients - I Chart

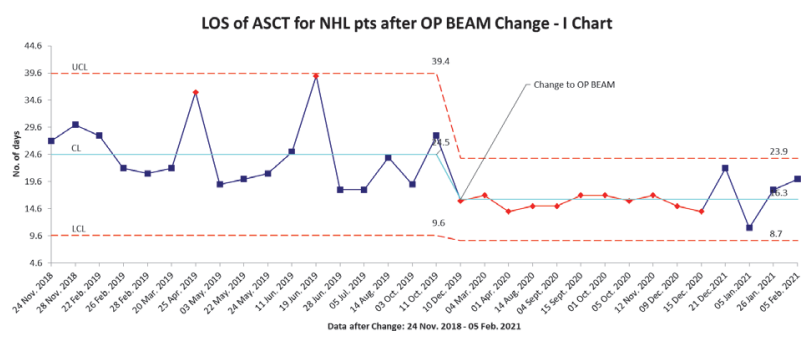

Abstract 8 Figure 5 LOS for ASCT for NHL patients after OP BEAM Change - I Chart
Conclusions Increase in the capacity for ASCT by decreasing the LOS by change of IP Conditioning Chemotherapy to OP setting is possible and feasible in QI framework.

\section{USING THE QI ESSENTIALS TOOLKIT TO ASSESS RISK, DRIVE AND SUSTAIN IMPROVEMENT IN MEDICATION SAFETY}

Mouna Ayoub Bahsoun, Anya Neidig. Intermountain Primary Children's Hospital

\subsection{6/bmjoq-2021-|HI.9}

Background Barcode-assisted medication administration (BCMA) is an evidence based practice to reduce medication administration errors; however, compliance rates with the use of this technology sometimes vary between different healthcare workers. Improving BCMA compliance benefits healthcare organizations in their efforts towards Zero Harm.

Objectives Achieve less than 2\% BCMA overrides at a pediatric hospital, within a 12 months' timeframe.

Methods To improve BCMA adherence across all disciplines, the IHI's QI Essentials Toolkit was used to guide an

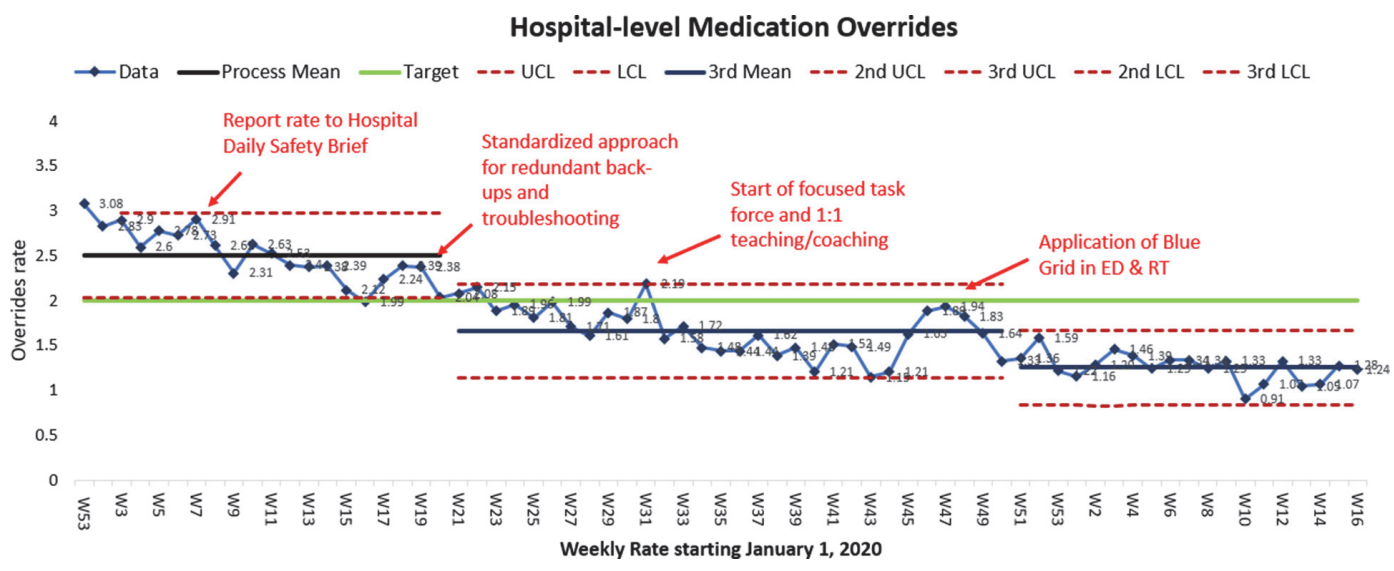

Abstract 9 Figure 1 Hospital-wide medication overrides 